\title{
The Improvement of the Region Homogeneity Measure Operator and Its Noise Disposed
}

\author{
Senyue Luo \\ Department of Basic Education, Guangdong Radio \& TV University, Guangzhou, Guangdong 510091, China \\ Correspondence should be addressed to Senyue Luo; syluo@gdrtvu.edu.cn
}

Received 11 January 2014; Revised 19 March 2014; Accepted 20 March 2014; Published 28 April 2014

Academic Editor: Erik Cuevas

Copyright (C) 2014 Senyue Luo. This is an open access article distributed under the Creative Commons Attribution License, which permits unrestricted use, distribution, and reproduction in any medium, provided the original work is properly cited.

Based on the analysis of the characteristic of the image texture, we extend the region homogeneity measure operator (RHMO) to a more reasonable case, thus avoiding some omission situation. Moreover, we compare the RHMO with the convolution function to find out the more rational range of region homogeneity measure (RHM). At the end of this paper, we use the improved RHM method for some images in a new Partial Differential Equation (PDE); the results indicate that the improved concept has achieved a good result.

\section{Introduction}

In the recent thirty years, various digital image processing methods based on PDE [1-3] have been put forward and studied widely. These methods will overcome some defects of the classical image processing in some aspects, so they have attracted the attention of numerous researchers and have been applied to image processing widely.

In the field of classical image processing [4], Fourier method can be regarded as the main mathematical method. In the algorithm of the classical image processing, the smooth method has a serious defect: while doing the image smooth processing, it will also do the same processing on the edge of the image, which is a main characteristic of an image. So after smoothness, some characteristics of edge have been eliminated. Generally speaking, the traditional methods in image processing are difficult to achieve the ideal effect. But the digital image processing method which is based on PDE has overcome the difficulty described above to some degree.

Different from the traditional method, the RHMO [5] does not use the gray gradient which is defined among several pixel points. Instead, the gradient is replaced by the average gray gradient which includes all the pixel points in the neighborhood. This simple replacement can achieve a similar effect in image processing as the traditional method. RHM is defined as follows.
Definition 1 (RHM). Let $B(P, r)$ be a circle in which the center is $P=\left(x_{0}, y_{0}\right)$ and the semidiameter is $r$. The angle between $x$-axis and $r$ is $\theta$. Diameter $\ell$ divides $B(P, r)$ into two parts: $S_{1}(\theta)$ and $S_{2}(\theta)$, and we write

$$
\begin{gathered}
U\left(\theta, r ; x_{0}, y_{0}, t\right) \\
=\left|\int_{S_{1}(\theta)} u(x, y, t) d x d y-\int_{S_{2}(\theta)} u(x, y, t) d x d y\right|, \\
R_{L} u=\bar{U}_{L}\left(x_{0}, y_{0}, t\right)=\max _{0 \leq \theta \leq \pi} \sup _{|r| \leq L} U\left(\theta, r ; x_{0}, y_{0}, t\right) ;
\end{gathered}
$$

then, we define $R_{L} u$ as the RHM of $u$ and $R_{L}$ as the RHMO of $u$.

In (1), the noise's characteristics of single pixel and region have been considered. As to the single pixel, we are very approximate to regard it as a solid circle (as Figure 1). While dividing the area, if the diameter $\ell$ passes through $P$ which is the center of a circle, the value of $R_{L}$ is zero. On the other hand, because the distribution of the noise is random, in all whirling directions of $\ell$, the distribution and the intensity of the noise are all the same on probability (as Figure 2). The influence of the noise is counteracted. So the value of $R_{L} u$ will not change obviously - while its value can reflect conformity of area's characteristic. At the same time, the value can reflect the possibility of the image's edge. 


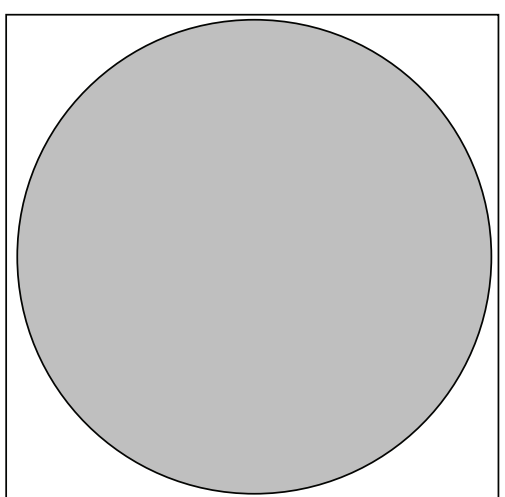

FIgURE 1

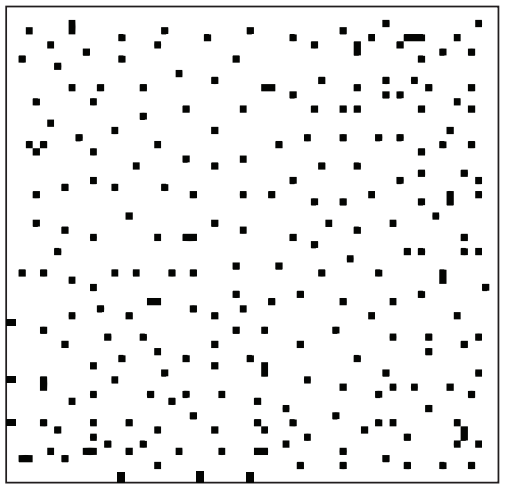

FIGURE 2

In the subsequent part of this paper, we improve the RHMO by the analysis of the characteristic about the noise and the texture, thus preventing some situations that RHMO will ignore in the processing of the image. On the other hand, we provide another improvement of RHMO through the analysis of the convolution term and the RHMO term and that lead to a more reasonable range of the value of RHMO.

\section{The Improvement of the Model}

2.1. The New Division for the Region. During the process of calculating RHM, choosing the semidiameter $L$ depends on the characteristic of the image. If the distribution of the edge is dispersed, the value of $L$ can be a bigger one; for the opposite situation (intensive situation), the value of $L$ should be a smaller one. But the treatment may be insensitive to some particular texture. So we may regard the corresponding texture as the noise to deal with. We consider such an area (as Figures 3 and 4 show, the distribution of texture has axial symmetry property) according to the definition of RHMO at this moment; we regard the texture as the noise to deal with. This kind of situation may appear, for example, in the picture of fingerprint, and an area of $20 \times 20$ pixels may distribute 2 or 3 strips of fingerprint. Of course, we can break the symmetry of Figures 3 and 4 by dwindling the value of $L$; thus the texture of the image can be searched out. Because we cannot distinguish the noise and true texture in a small region, the value of $L$ cannot be too small (especially in this situation, the semidiameter of the noise is bigger than the normal size).

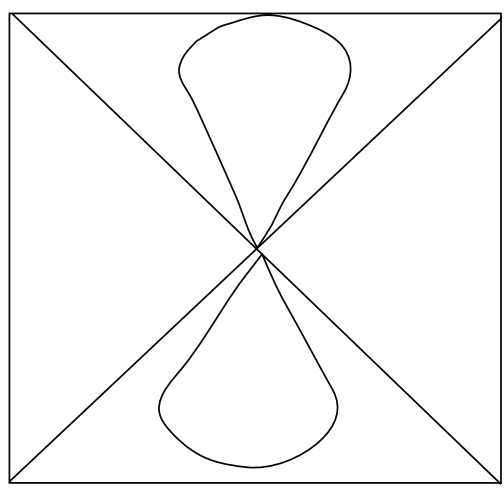

Figure 3



FIGURE 4

In fact, we can use a new definition about the RHM which does not calculate the difference between two areas divided by the diameter. We can make a series of diameters through the center of a circle; the angles between the two adjoint semidiameters are equal to each other. Then these small partitions (for the square region, these partitions are triangles) are divided into two regions where the areas are equal to each other. At the end, we can get the minimum absolute value of these two pieces' difference. For example, we can cross the center of the region to make two vertical diameters (such as Figures 3 and 4) and get the valve by the steps mentioned above. From Figures 3 and 4, by the improvement method, we can calculate the RHM's value: just the sum of two triangular regions from head to foot deducts the sum of the other two triangular regions. But according to the algorithm mentioned first, the value of RHM will be zero, so the texture of the picture will be ignored as noise.

Of course, dividing the region with several parallel straight lines will also be a working method (as Figure 5). We can do this step first and then make up them into two regions to get the RHM's value. But each small region for summing cannot be equal to each other, because in each small region the area of the noise must be equal to each other. Compared with the previous method, this division is more difficult to achieve. Hence, we adopt the first kind of method to define RHM.

Therefore, we provide the following definition of the improved RHM. 


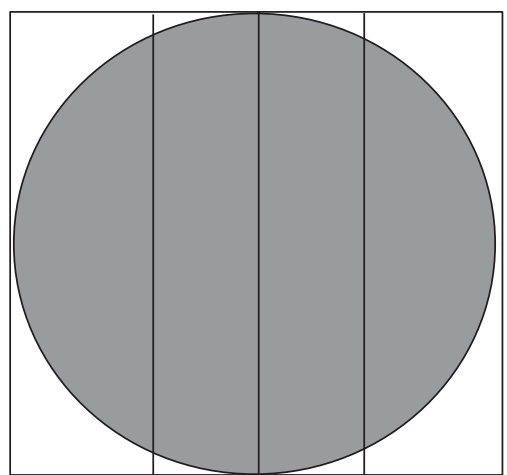

FIgURE 5

Definition 2 (the improvement RHM). Let $B(P, r)$ be a circle in which the center is $P=\left(x_{0}, y_{0}\right)$ and the semidiameter is $r$. Set $n / 2$ as integer. The diameters $l_{1}, l_{2}, l_{3}, \ldots, l_{n}$ divide $B(P, r)$ into $2 n$ part $\left(\theta_{1}, \theta_{2}, \theta_{3}, \ldots, \theta_{2 n}\right)$, and the minimum angles which are divided by these diameters are equal to each other, and these $2 n$ partitions make up two equal parts in area without repeated $S_{1}$ and $S_{2}$. The angle between $x$-axis and $l_{1}$ is $\theta$, and we write

$$
\begin{gathered}
U_{1}=\mid \int_{S_{1}\left(\theta_{1}+\theta_{2}+\cdots+\theta_{n}\right)} u(x, y, t) d x d y \\
-\int_{S_{2}\left(\theta_{n+1}+\theta_{n+2}+\cdots+\theta_{2 n}\right)} u(x, y, t) d x d y \mid ; \\
\quad-\mid \int_{S_{1}\left(\theta_{1}+\cdots+\theta_{n / 2}+\theta_{n+1}+\cdots+\theta_{3 n / 2}\right)} u(x, y, t) d x d y \\
\int_{S_{2}\left(\theta_{(n / 2)+1}+\cdots+\theta_{n}+\theta_{(3 n / 2)+1}+\cdots+\theta_{2 n}\right)} u(x, y, t) d x d y \mid,
\end{gathered}
$$

then, we define $R_{L} u$ as the improved RHM of $u$ and $R_{L}$ as the improved RHMO of $u$.

2.2. The Adjustment for the RHM. In the field of digital image processing, the anisotropic diffusion model which is set up by Perona and Malik [1] is a typical one and used widely. The model replaces the gauss smoothing with coefficient directionality distribution, so it arouses the attention of numerous researchers. A kind of nonlinear parabolic PDE used for image processing has been put forward [3] by on the basis of the forefathers:

$$
\begin{gathered}
\frac{\partial u(x, y, t)}{\partial t}=g\left(\left|\nabla G_{\sigma} * u\right|\right)|D u| \operatorname{div}\left(\frac{D u}{|D u|}\right), \\
u(x, y, 0)=u_{0}(x, y) .
\end{gathered}
$$

$u_{0}(x, y)$ is the grey gradient of the primitive image. $u(x, y, t)$ is the image when it is at time $t . G$ is a smooth kernel (e.g., gauss smooth kernel). $G * D u$ is the local estimation of the gradient $|D u| . g(s)$ is the nonincreasing real function, while $|\cdot|_{s} \rightarrow \infty, g(s)$ tends to 0 . This degenerative model treats the image with the alternative smooth method. In this processing, the edge of the image is maintained while the other place of it becomes smooth.

In this paper, we couple the Alvarez model locally with a two-order PDE. Furthermore, we combine the HRMO with this coupling processing; it leads to a new PDE model of anisotropic diffusion. Exactly, we substitute the $\left|\nabla G_{\sigma} * u\right|$ term with the RHM term $\left(R_{L} u\right)$.

Through rough estimation, as to a $21 \times 21$ pixels region (such as a region of a bmp image of 256 grade), the range of $R_{L} u$ is from 0 to $256 \times 21 \times 10$. This range of the value may cause a relatively large margin in $g\left(R_{L} u\right)$ term in the PDE. But the function of $g\left(R_{L} u\right)$ is used for strengthening the edge of the image. In fact, the $g\left(R_{L} u\right)$ term has controlled the pace spread in the image processing; the value of $g\left(R_{L} u\right)$ is in inverse proportion to rate of diffusion. This may lead to the fact that the image disposed may show a very different effect in each region after the diffusion of the image (in the keeping of the edge). So we consider to adjust the value field of $R_{L} u$.

The method we can use is through comparing the convolution function with $R_{L} u$ term in the value. As to the convolution term $\left|\nabla G_{\sigma} * u\right|$, the value field is far little than the range of $R_{L} u$ (the detailed reason can be seen in the following analysis). But it is difficult to define the convolution term $\left|\nabla G_{\sigma} * u\right|$. So we can consider the method used in [2], that is, using the $\left|\nabla G_{\sigma}(u ; x, y, t, \sigma)\right|$ to replace $\left|\nabla G_{\sigma} * u\right|$.

To the arbitrary $u(x, y, t) \in C\left([0, T] ; L^{2}(\Omega)\right)$, we have

$$
\begin{aligned}
& G_{\sigma}(u ; x, y, t) \\
& =c_{0}(t)+\sum_{i} \sum_{j} c_{i j}(t) e^{-\left[(i \pi / b)^{2}+(j \pi / a)^{2}\right] \sigma} \\
& \quad \times \cos \left(\frac{j \pi}{a} x\right) \cos \left(\frac{i \pi}{b} y\right) .
\end{aligned}
$$

As to $c_{0}(t), c_{i j}(t), u$ mentioned above, we have the following Fourier expansion:

$$
u(x, y, t)=c_{0}(t)+\sum_{i} \sum_{j} c_{i j}(\mathrm{t}) \cos \left(\frac{j \pi}{a} x\right) \cos \left(\frac{i \pi}{b} y\right) .
$$

By combining (5) with (4), we can get $G_{\sigma}$. Then we can calculate the value of $\left|\nabla G_{\sigma}(u ; x, y, t, \sigma)\right|^{2}$. So we can provide the restrain coefficient; that is,

$$
\lambda=\frac{\max \left\{R_{L} u\right\}}{\max \left\{\left|\nabla G_{\sigma}(u ; x, y, t, \sigma)\right|^{2}\right\}} .
$$

In fact, we can provide a roughness estimation about the value of $\lambda$. By calculating a two-dimensional discrete convolution $H=F \times G$ in a $3 \times 3$ pixels area,

$$
H(i, j)=\sum_{m} \sum_{n} F(m, n) G(i-m, j-n),
$$

where $i, j$ represent the concrete pixel of a picture; $G, F$, respectively, represent the convolution kernel and image. As to convolution kernel, we can let

$$
G=\left[\begin{array}{ll}
-1 & 1 \\
-2 & 2
\end{array}\right]
$$


for the matrix

$$
F=\left[\begin{array}{ll}
1 & 2 \\
3 & 4
\end{array}\right]
$$

the convolution of them

$$
H=F * G=\left[\begin{array}{ccc}
-1 & -1 & 2 \\
-5 & -3 & -8 \\
-6 & -2 & -8
\end{array}\right]
$$

As to a $3 \times 3$ pixels region of bmp image which is 256 grade, the absolute value of the sum of the matrix's element which is given by the convolution kernel is $0 \sim 256 \times 9$ (consider the sum between the negative and positive elements of the matrix). So we can get the coefficient of the restraining; that is, $\lambda=(256 \times 21 \times 10) /(256 \times 9) \approx 23$.

\section{Setting-Up of the Model}

From the above-mentioned analysis, combining with (2), we will propose the improvement model which comes from the Alvarez PDE model (3). It can overcome some shortcomings of the original model and get better result while handling the noise problem.

Let $\Omega$ be an open set of $\mathfrak{R}^{2}$; let $\Gamma$ be its smooth border. Consider the following initial-boundary value problem:

$$
\begin{gathered}
\frac{\partial u(x, y, t)}{\partial t}=g\left(\frac{1}{\lambda} R_{L} v\right)|D u| \operatorname{div}\left(\frac{D u}{|D u|}\right), \\
u(x, y, 0)=u_{0} .
\end{gathered}
$$

Similar to Alvarez model, $u_{0}$ in (12) is the initial signal, $g: R^{+} \rightarrow R^{+}$is a decreasing function, and $g(0)=$ 1 , $\lim _{t \rightarrow+\infty} g(t)=0$. Let $g(t)=1 /\left(1+t^{2}\right)$; then $t \rightarrow g(t)$ is smooth. The difference is as follows: in the model, $R_{L} u$ is the RHM and $u(x, y, t)$ is the unique solution of the following linear questions (achieving the same effect as the convolution function [6]):

$$
\begin{gathered}
\frac{\partial v(x, y, t)}{\partial t}-\Delta v=0, \\
\left.v(x, y, t)\right|_{t=0}=v_{0}(x, y) .
\end{gathered}
$$

Through choosing the appropriate threshold value $L$ and solving the equation above to get the solution $u(x, y, t)$, we can get the sharp image and the clear border of the image.

\section{Numerical Scheme for the Model}

Suppose that $\Omega$ is the $M \Delta h * N \Delta h$ rectangle area where $\Delta h$ is the unit length. For example, the side of the rectangle neighborhood is $L=20 \Delta h$. We can write

$$
\begin{gathered}
u_{i, j}^{n}=u(i \Delta h, j \Delta h, n \Delta t), \\
\sum \sum_{(i, j) \in S_{1}(\theta)} u_{i, j}^{n}=\int_{S_{1}(\theta)} u(x, y, t) d x d y .
\end{gathered}
$$

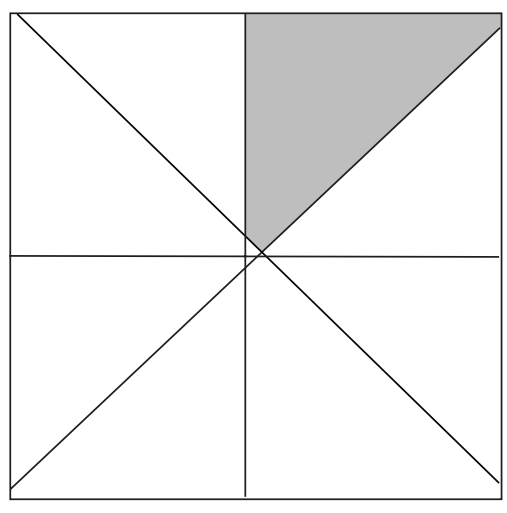

Figure 6

For the situation of double diameter, when the diameter whirl in $\theta \in\left[0^{\circ}, 180^{\circ}\right]$ one time and $h \in[0, L]$, we have

$$
\begin{aligned}
& U_{1}(\theta, h ; x, y, t) \\
& =\left\{\begin{array}{c}
\left|\sum_{i=-10}^{10} \sum_{j=[i \tan \theta]+1}^{10} u_{i, j}^{n}-\sum_{i=-10}^{10} \sum_{j=-10}^{[i \tan \theta]} u_{i, j}^{n}\right|, \\
\theta \in\left[0^{\circ}, 45^{\circ}\right] \cup\left[135^{\circ}, 180^{\circ}\right] ; \\
\left|\sum_{j=-10}^{10} \sum_{i=[j \tan \theta]+1}^{10} u_{i, j}^{n}-\sum_{j=-10}^{10} \sum_{i=-10}^{[j \tan \theta]} u_{i, j}^{n}\right| \\
\theta \in\left[45^{\circ}, 135^{\circ}\right] .
\end{array}\right. \\
& U_{2}(\theta, h ; x, y, t) \\
& \left.=\mid 2\left(\sum_{i=-10}^{[-10 \tan \theta]} \sum_{j=[i \tan \theta]}^{10}+\sum_{i=[-10 \tan \theta]+1}^{10} \sum_{j=-10}^{[i \tan \theta]} u_{i, j}^{n}\right)\right)-U_{\text {sum }} \mid,
\end{aligned}
$$

where

$$
\theta \in\left[0^{\circ}, 45^{\circ}\right], \quad U_{\text {sum }}=\sum_{i=-10}^{10} \sum_{j=-10}^{10} u_{i, j}^{n} ;
$$

furthermore

$$
R_{L} u(x, y, t)=\max _{\theta \in\left[0^{\circ}, 180^{\circ}\right]} \sup _{|h| \leq L}\left\{U_{1}, U_{2}\right\}
$$

For the situation of many diameters, we can also provide a similar definition by the formula given above. Consider the difficulty of realizing of the process; we can use the method which multiplies with matrix. For example, as to the four diameters situation, we can get the sum of the pixel of every triangle region through the multiplication of the matrix. Then calculate the RHM by the way of making up every triangle region at random (there are $C_{7}^{3}=35$ kinds of combination altogether).

The pixel's sum of shadow region in Figure 6 (a $5 \times 5$ pixels area) is (the sum of other regions can be similarly given)

$$
U_{\text {sum }}=\sum_{i=-2}^{2} \sum_{j=-2}^{2} u_{i, j}^{2} u_{i, j}^{n}
$$




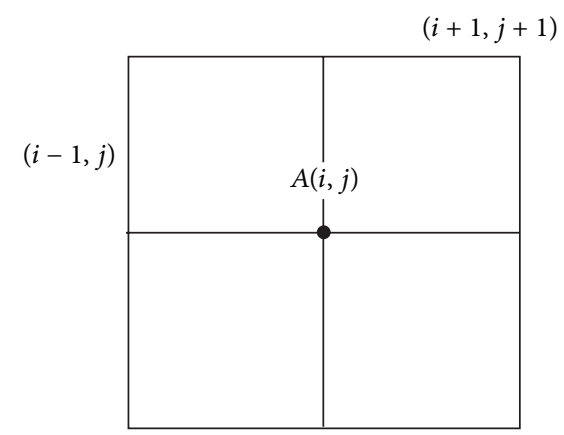

FIGURE 7



Figure 8
Use $\sum_{n=0}^{3} f_{n}\left(D u^{k} /\left|D u^{k}\right|\right)\left(\partial^{2} u^{k} / \partial x_{n}^{2}\right)$ to approximate $|D u| \operatorname{div}(D u /|D u|)$, and $\theta$ is the angle between each direction and $D u$; then $f_{n}$ can be defined as follows:

$$
f_{n}\left(\theta_{n}\right)= \begin{cases}1, & \left|\theta_{n}\right| \leq \theta_{0}, \\ 0, & \left|\theta_{n}\right| \geq \frac{4}{\pi}-\theta_{0}, \\ \left(\frac{4}{\pi}\right)^{4}\left(\left|\theta_{n}\right|-\frac{4}{\pi}+\theta_{n}\right)^{4}, & \text { else, }\end{cases}
$$

where $\theta_{0}$ is a constant. Using $\Delta u^{k}$ to stand for Laplace operator, the two-order differential coefficient about the four directions is $\partial^{2} u^{k} / \partial x_{n}^{2} \cdot \tau$ is step of the time. The difference equation about (11) is

$$
\frac{u^{k+1}-u^{k}}{\tau}=g\left(\frac{1}{\lambda} R_{L} u\right) \sum_{n=0}^{3} f_{n}\left(\frac{D u^{k}}{\left|D u^{k}\right|}\right)\left(\frac{\partial^{2} u^{k}}{\partial x_{n}^{2}}\right) .
$$

As to the (13), for obtaining better data precision, we can use the following implicit scheme-the solution of (13) meets the following Douglas-Rachford difference equation:

$$
\begin{aligned}
-2 l v_{i-1, j}^{n+(1 / 2)} & +(1+4 l) v_{i, j}^{n+(1 / 2)}-2 l v_{i+1, j}^{n+(1 / 2)} \\
& =2 l v_{i, j-1}^{n}+(1-4 l) v_{i, j}^{n}+2 l v_{i, j+1}^{n}, \\
-2 l v_{i, j-1}^{n+1} & +(1+4 l) v_{i, j}^{n+1}-2 l v_{i, j+1}^{n+1} \\
& =2 l v_{i-1, j}^{n+(1 / 2)}+(1-4 l) v_{i, j}^{n+(1 / 2)}+2 l v_{i+1, j}^{n+(1 / 2)},
\end{aligned}
$$

where $i=2, \ldots, M-1 ; j=2, \ldots, N-1 ; l=\Delta t / 4 \Delta h^{2}$ and

$$
\begin{array}{ll}
v_{i, 1}^{n+1}=v_{i, 2}^{n+1}, & v_{M-1, j}^{n+1}=v_{M, j}^{n+1}, \\
v_{1, j}^{n+1}=v_{2, j}^{n+1}, & v_{i, N-1}^{n+1}=v_{i, N}^{n+1},
\end{array}
$$

where $i \in\{1, \ldots, M\}, j \in\{1, \ldots, N\}$.

where

$$
U=F \times\left[\begin{array}{ccccc}
0 & 0 & 0 & 0 & 0 \\
0 & 0 & 0 & 0 & 0 \\
1 & 0 & 0 & 0 & 0 \\
1 & 1 & 0 & 0 & 0 \\
1 & 1 & 1 & 0 & 0
\end{array}\right]
$$

For the main equation of (11), we can use the $3 \times 3$ (as Figure 7) difference scheme.

When we calculate the diffusion operator $|D u| \operatorname{div}(D u /|D u|)$, we must do approximation on the mesh. Suppose that $A$ in which the coordinate is $(i, j)$ is the cross point of the mesh. Then we can define the four directions, respectively, as follows:

$$
\begin{gathered}
(i-1, j) \longrightarrow(i+1, j), \\
(i, j-1) \longrightarrow(i, j+1), \\
(i-1, j-1) \longrightarrow(i, j+1), \\
(i-1, j+1) \longrightarrow(j+1, j-1) .
\end{gathered}
$$

\section{Numerical Experiments and Results}

5.1. The Choice of the Parameters. In the experiment, we try to make the adjustment to algorithm time $(T)$ and length of stride (step), diligently making the balance in the computation load and the picture quality. Here, the processed pixels bmp images. The original image and image added to the gauss noise are, respectively, as the pictures below (Figures 8 and 9).

The left parts of Figure 10 Figure 15 that come from Figure 9 are given by the algorithm in this paper. The left part of Figure 10 is the denoising image of Figure 9, while the right side image is edge extraction of the left side image (using the canny operator). The edge extraction image may reflect the quality of denoising image-reflecting the retention of the edge after the denoising. Figure 11 Figure 15 are also presented in the same way. Following the concrete result, we analyze the following.

(1) The $T$ of Figure 10 Figure 13 is $1 \mathrm{~s}$, while the step of each other, respectively, is $0.25 \mathrm{~s}, 0.05 \mathrm{~s}, 0.02 \mathrm{~s}$, and objects which are brain's MRI images are all for $128 \times 128$ 


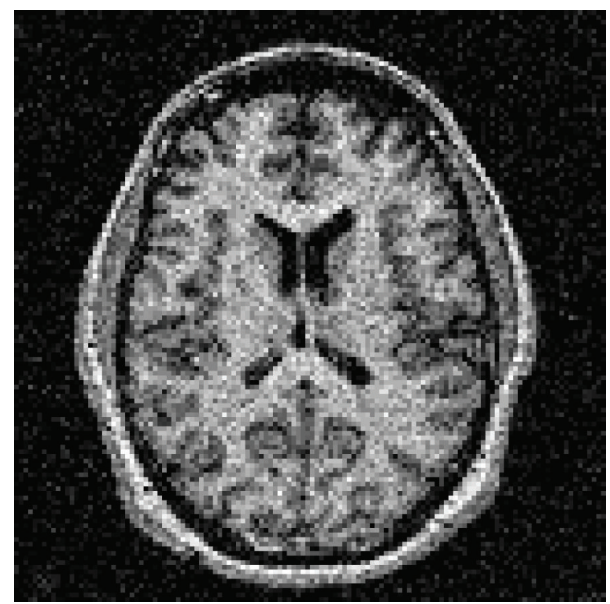

Figure 9
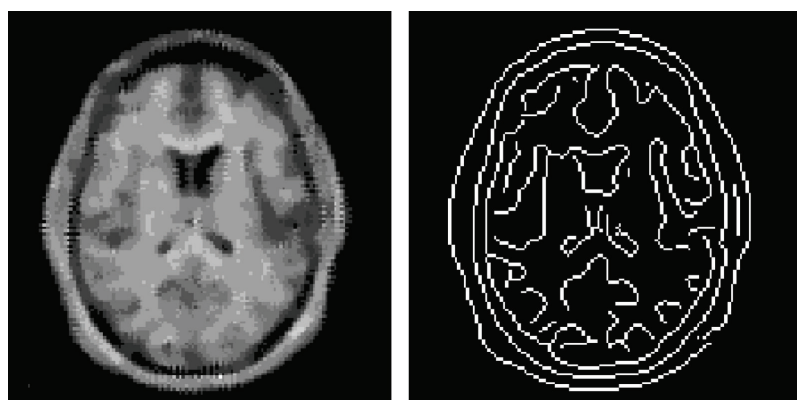

Figure 10
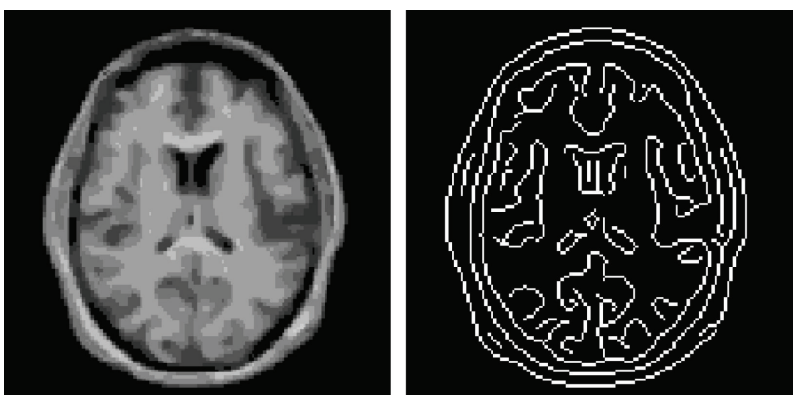

FigURE 11
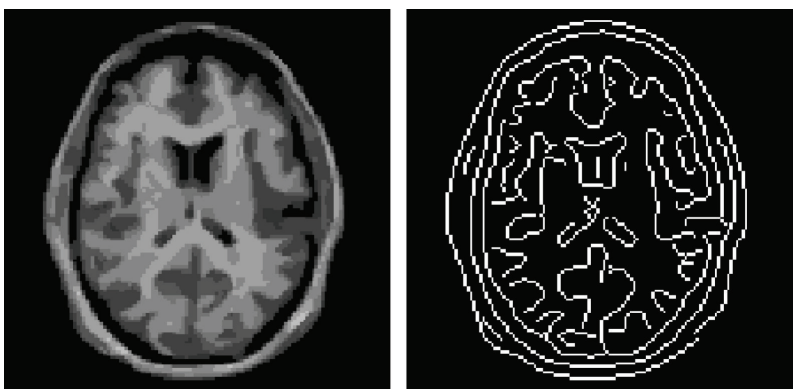

Figure 12
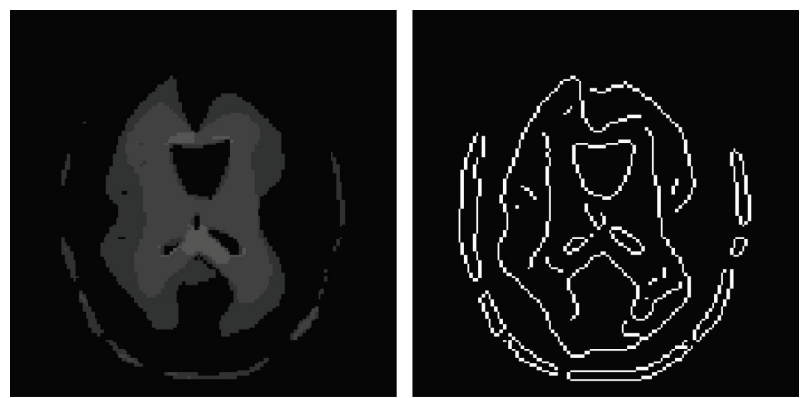

FIGURE 13


FIGURE 14

0.005 s. Figure 10 completely eliminates background noise but simultaneously also blurs many gyrus (possibly seen from its right side edge extraction image); the image in Figure 13 also has this kind of problem; comparatively the images in Figures 11 and 12 have obtained the good effect in the image denoising and the edge extraction; the step times of Figures 11 and 12 are, respectively, 20 and 50; however, some details of the texture will not be kept; for instance, we can see from Figures 11 and 12 that some details of gyrus are lost.

(2) The $T$ of Figure $14 \sim$ Figure 15 all is $0.005 \mathrm{~s}$, while the step of each, respectively, is $0.0001 \mathrm{~s}$ and $0.00005 \mathrm{~s}$; the two images eliminate background noise well; moreover, two images achieve a more ideal effect in the details maintained; comparatively, Figure 14 is clearer than Figure 15 (this can be seen by comparing it with the original image in Figure 8); moreover, the step times of Figure 14 are 50, while of Figure 15 are 100; therefore, considering the denoising effect and the algorithmic load, Figure 14 surpasses obviously Figure 15.

Considering the above two kinds of situations ( $T$ and step), in order to get the high image denoising quality, we may use the $T$ and step which Figure 14 uses; but in the situations in which image quality request is not high and computation real-time request is high, the scheme which Figure 11 uses can be considered.

5.2. Comparison of the Models. We will present some numerical results of the different models by the computer simulation. 

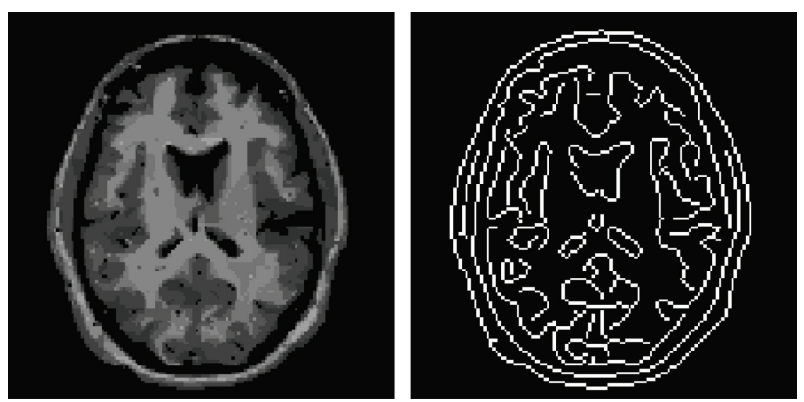

Figure 15

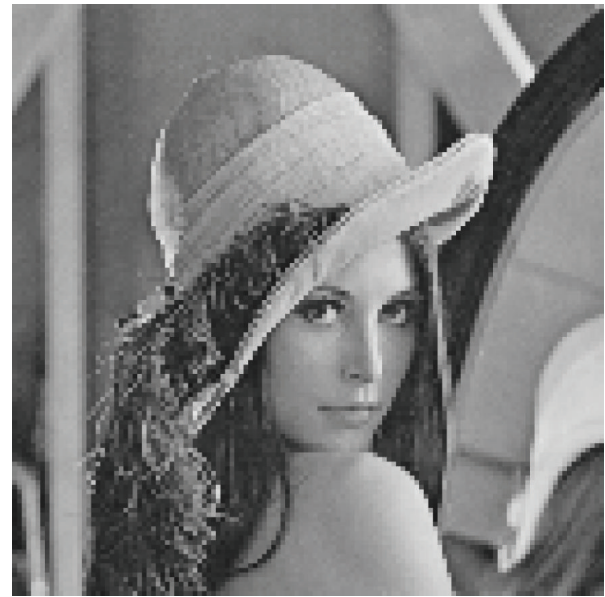

FIGURE 16

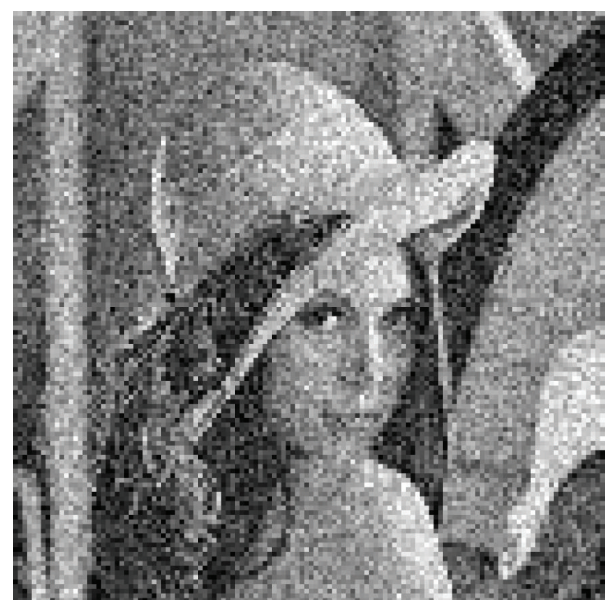

Figure 17

The below images of Lena are all for $128 \times 128$ pixels bmp images. Among the images, Figures 16 and 17 are, respectively, the original image and the image added with gauss noise. We also use the canny to get the edge of the images.

Figure 18 Figure 20 are the edge extraction images after the image filtering (resp., convolution filtering, median filtering, and Wiener filtering). From Figures 18 and 19, we can see that some details of Figure 17 are lost. For example, in Figures 18 and 19, the mouth of Lena has been lost while the edge of face and background kelp well. From Figure 20, we

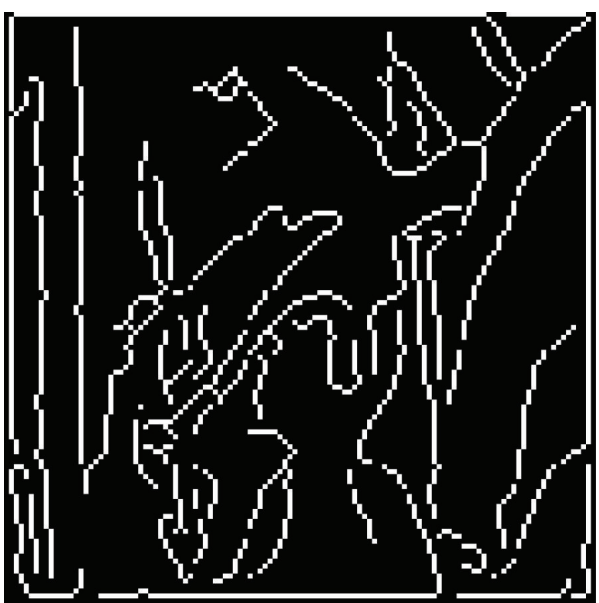

FIGURE 18

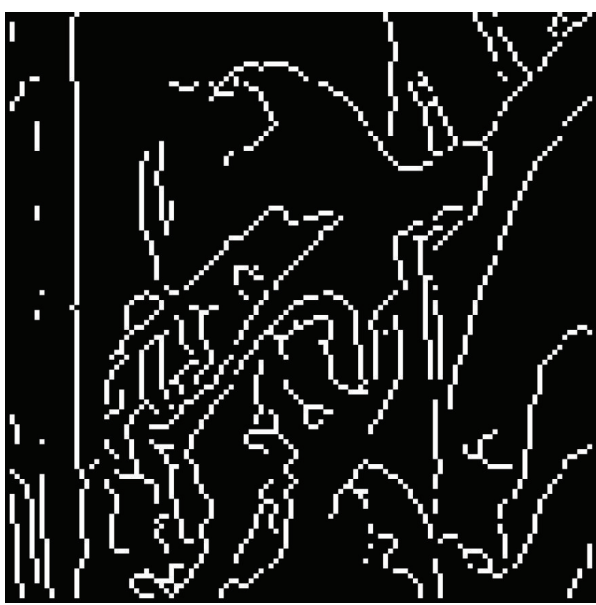

Figure 19

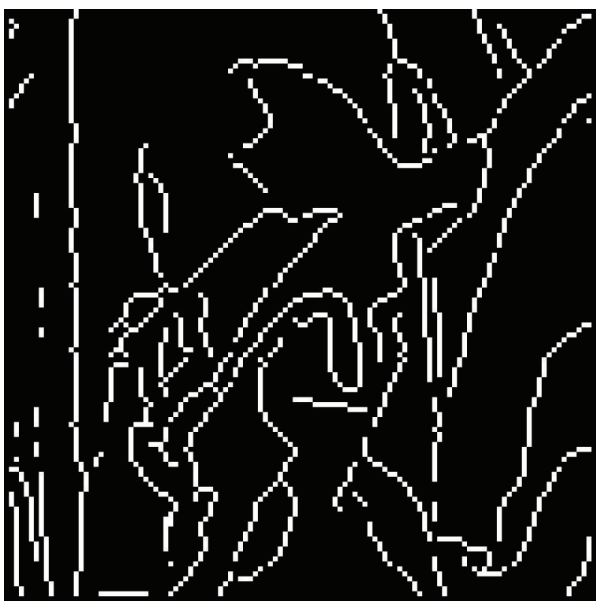

FIGURE 20

see that the edge of Lena's face is clear while many details of the background have been lost. In fact, the background is too simple after the diffusion.

Figures 21 and 22 are, respectively, the results of the original model and improved model. Two of them are better than Figure 18 Figure 20 in keeping the details of the original 


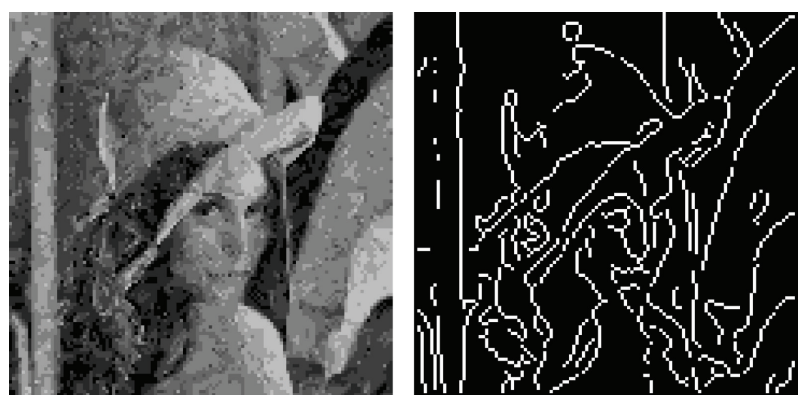

FIGURE 21

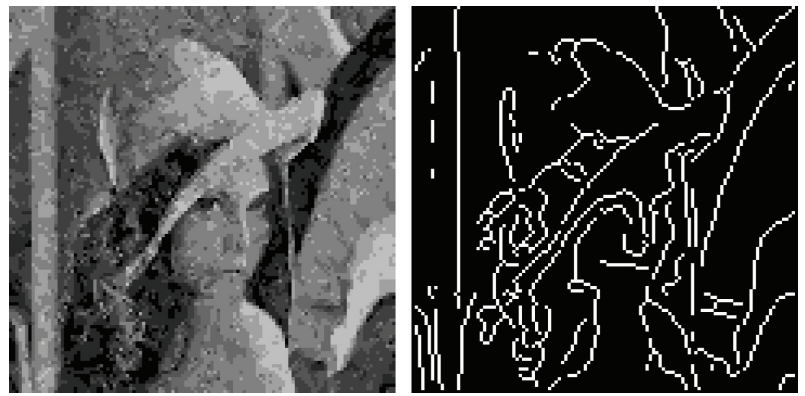

Figure 22

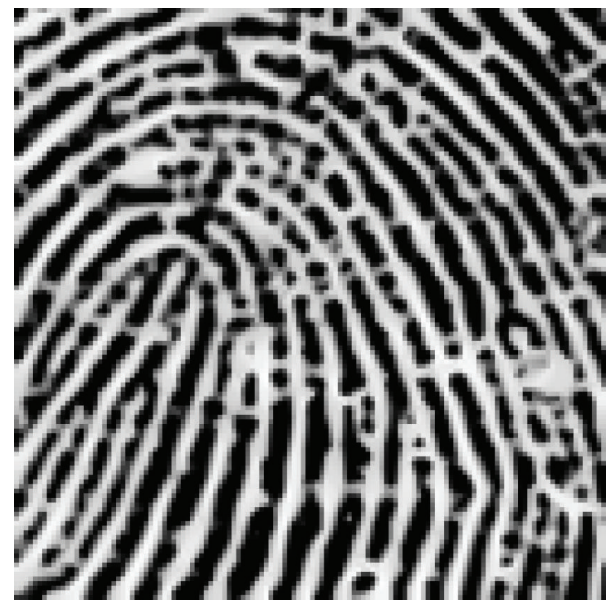

FIgURE 23

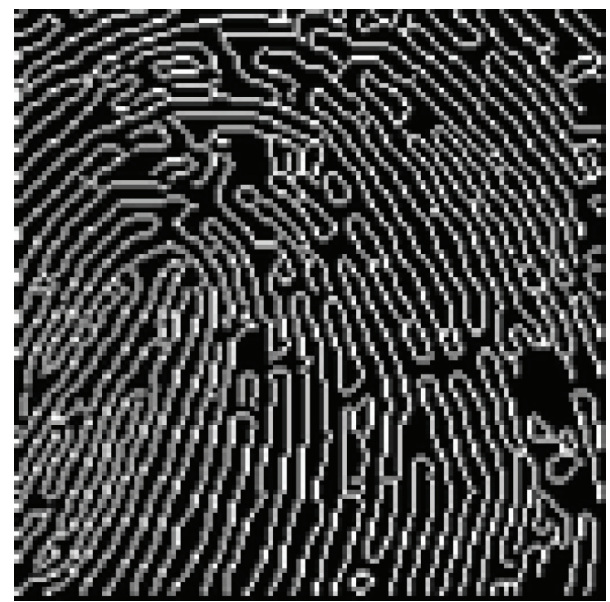

FIGURE 24

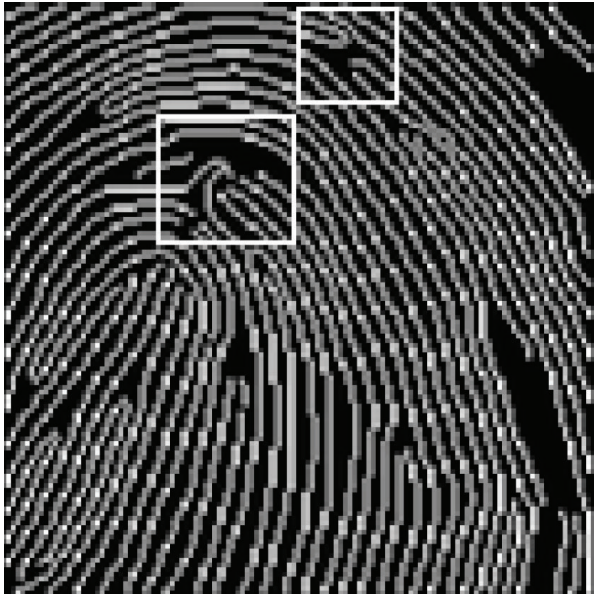

Figure 25



FIgURE 26

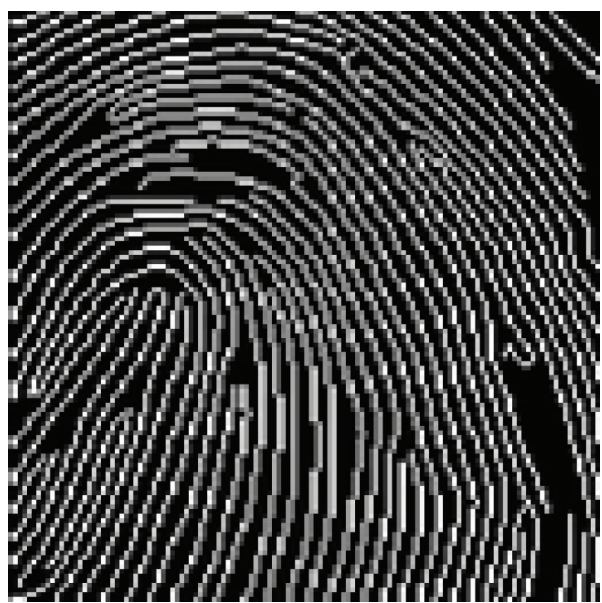

FIgURE 27

image. The difference of filtering images in Figure 17 is hard to be seen from the images. But when we resort to the edge images, we can see that Figure 22 is better than Figure 21 in denoising, while they all keep the edge well.

Because we cannot distinguish the noise and true texture in a small region, the value of $L$ cannot be too small. We 
choose a semidiameter $L=10$ during the process of calculating the picture of fingerprint. In this setting, the RHM will not deal with the texture of the fingerprint as the noise. So the most valuable texture will be retained. Figures 23 and 24 are the noising fingerprint and the contours of it.

In order to verify the effectiveness of our model, three different methods which include P-M model, Alvarez model, and improved RHM model are compared with experiments. In Figure 25 Figure 27, $T=0.004$, while the step of each other, respectively, is $0.0001 \mathrm{~s}$. The level set which comes from the diffusion processing of P-M model can be seen in Figure 25. In Figures 26 and 27, we deal in Figure 23 with the Alvarez model and improved RHMO method, respectively. From Figure 25 Figure 27, we can find out some subtle differences in the contours. In fact, we can find that the P-M model and Alvarez method form some false lines structures in local fingerprint information, while improved RHM method can achieve a good enhanced image, without generating false lines structure. This shows that our model can deal with more complex situations.

\section{Conclusions}

In this paper, we do some further researches about RHM based on the analysis of the RHM operator and the property of the image texture. We establish the new model by coupling the Alvarez model locally with a two-order PDE. In the new model, we substitute the $\left|\nabla G_{\sigma} * u\right|$ term with the RHM term $\left(R_{L} u\right)$. This model is a new PDE model of anisotropic diffusion. From the convolution operator, we deduce a rational range of the coefficient of RHM in our new PDE model. We test our model by the numerical method. From the experiment of brain's MRI images, we select the different algorithm time and length of stride to achieve a more reasonable diffusion effect of the picture. Comparing the result with some classic models and the original RHM model, we are convinced that our model achieves a good result in the diffusion of subtle texture.

\section{Conflict of Interests}

The author declares that there is no conflict of interests regarding the publication of this paper.

\section{References}

[1] P. Perona and J. Malik, "Scale-space and edge detection using anisotropic diffusion," IEEE Transactions on Pattern Analysis and Machine Intelligence, vol. 12, no. 7, pp. 629-639, 1990.

[2] F. Catte, P.-L. Lions, J.-M. Morel, and T. Coll, "Image selective smoothing and edge detection by nonlinear diffusion," SIAM Journal on Numerical Analysis, vol. 29, no. 1, pp. 182-193, 1992.

[3] L. Alvarez, P.-L. Lions, and J.-M. Morel, "Image selective smoothing and edge detection by nonlinear diffusion. II," SIAM Journal on Numerical Analysis, vol. 29, no. 3, pp. 845-866, 1992.

[4] A. P. Witkin, "Scale-space filtering," in Proceedings of the International Joint Conference on Artificial Intelligence (IJCAI '83), pp. 1019-1021, Karlsruhe, Germany, 1983.
[5] S. Fang, L. Jin, C. Guo, and R. Chen, "Region homogeneity measure and image enforcement model," in Proceedings of the $3 \mathrm{rd}$ International Congress on Image and Signal Processing (CISP '10), vol. 2, pp. 758-761, October 2010.

[6] K. Marr and E. Hilbreth, "Theory of edge detection," Proceedings of the Royal Society of London B, pp. 187-217, 1980. 


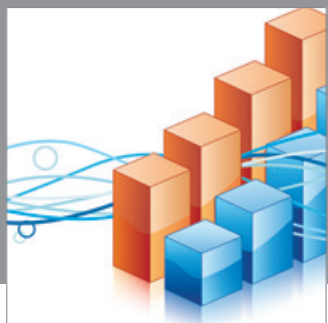

Advances in

Operations Research

mansans

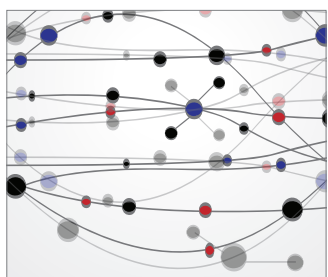

The Scientific World Journal
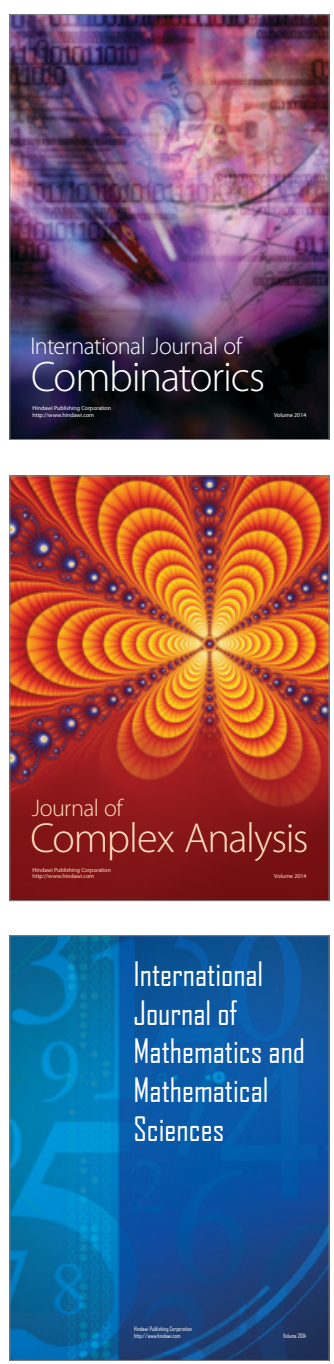
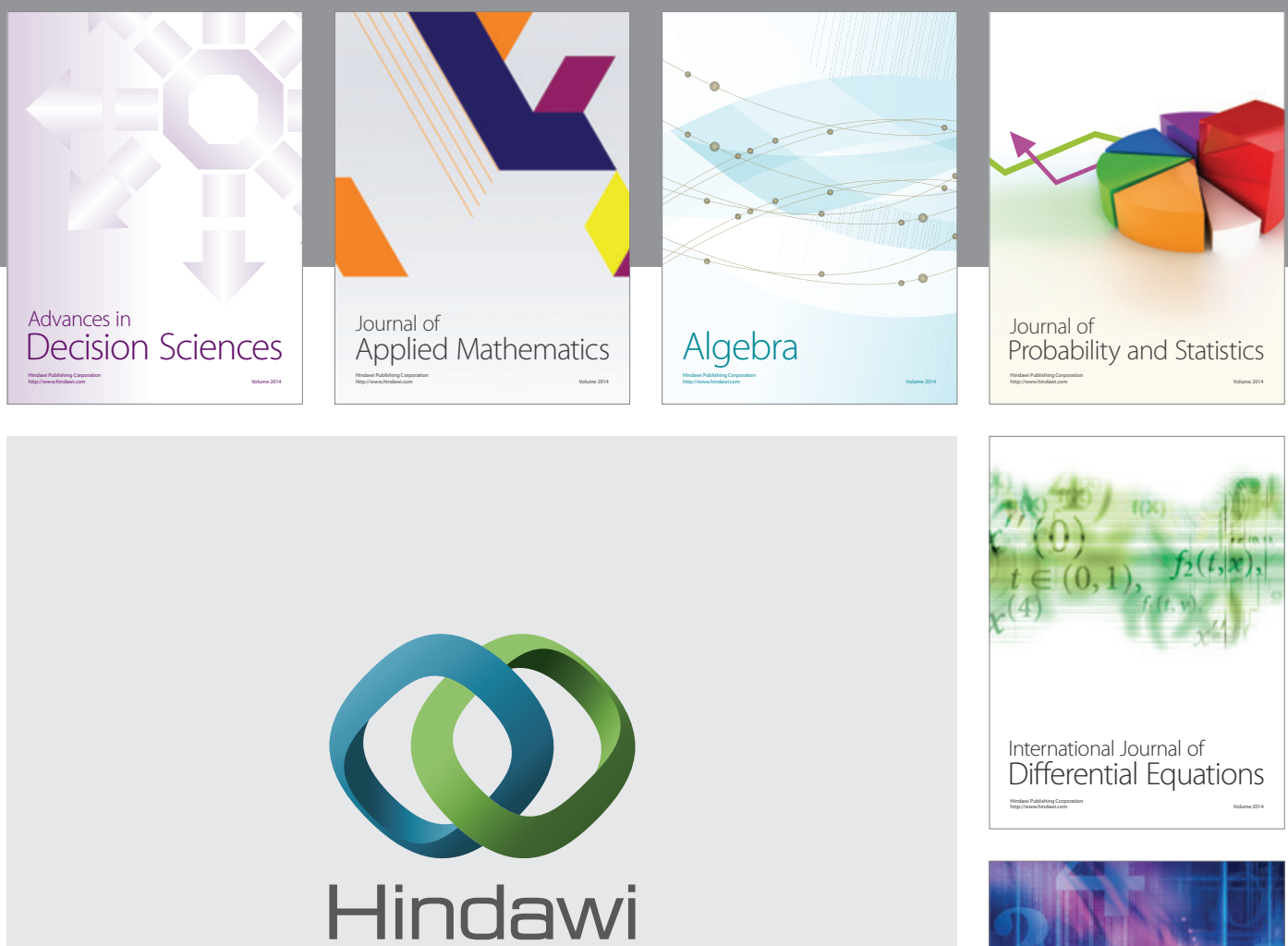

Submit your manuscripts at http://www.hindawi.com
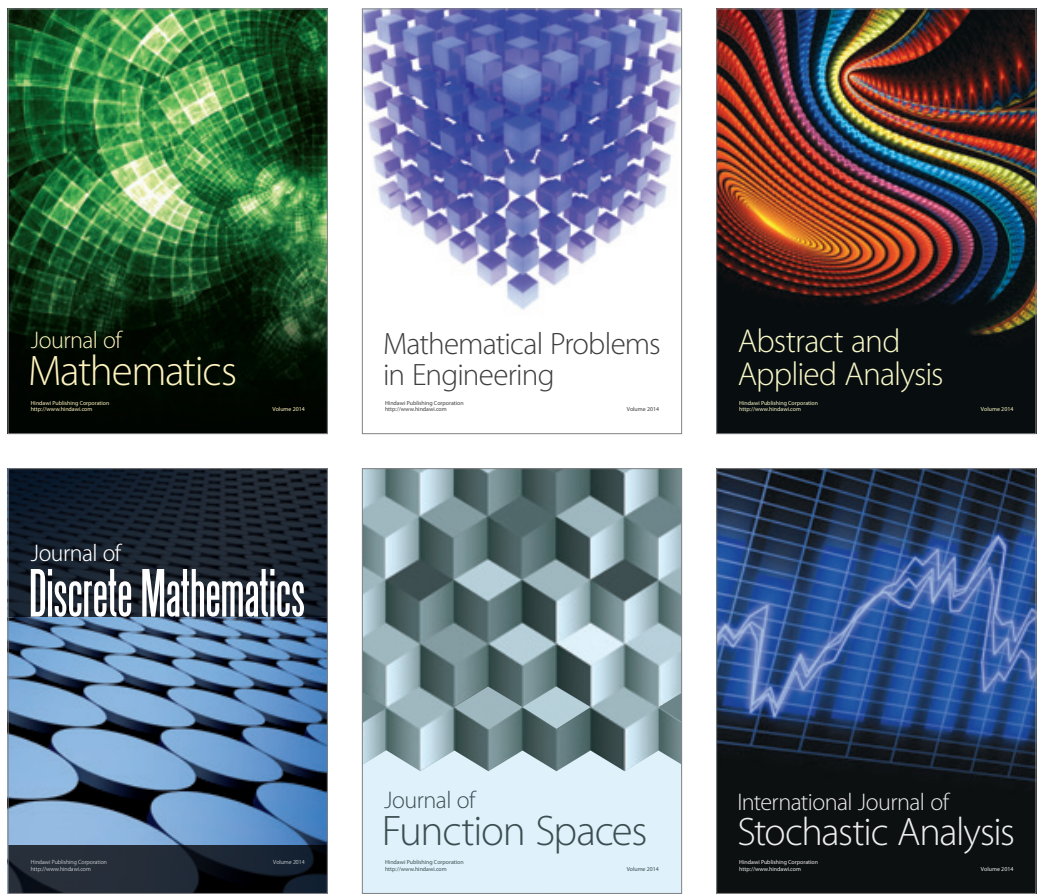

Journal of

Function Spaces

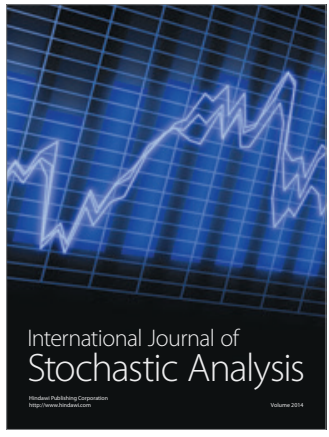

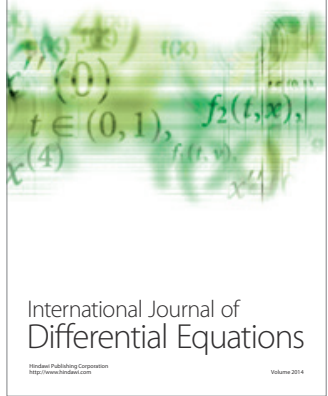
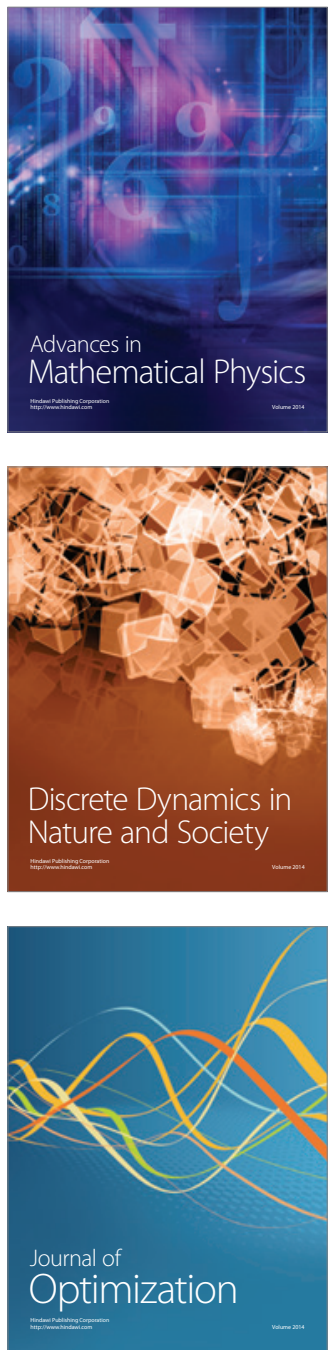